\title{
IS COLOR PERCEPTION OF PACKAGES AFFECTED BY THEIR IN-AISLE POSITION?
}

\author{
${ }^{1}$ Daniele Porcheddu, ${ }^{2}$ Maura Pugliatti and ${ }^{3}$ Antonio Pinna \\ ${ }^{1}$ Department of Economics and Business, \\ University of Sassari, Via Muroni 25, 07100 Sassari, Italy \\ ${ }^{2}$ Department of Clinical and Experimental Medicine and Oncology, \\ Section of Clinical Neurology, University of Sassari, Viale San Pietro 43, 07100 Sassari, Italy \\ ${ }^{3}$ Department of Surgery, Microsurgery and Medicine, \\ Section of Ophthalmology, University of Sassari, Viale San Pietro 43, 07100 Sassari, Italy
}

\begin{abstract}
The retail environment is characterized by numerous competing stimuli vying for the consumer's attention. Products packages feature both verbal and nonverbal elements. Nonverbal stimuli, like package colors, seem to be particularly relevant for perception. Knowing whether there is preferential positioning of colored items on the shelves is fundamental for retailers. In addition, some colors may be better perceived than others. The purpose of this study was to investigate whether the color perception of packages in a supermarket is affected by their in-aisle position and by their color type. In a lab setting, 120 right-handed subjects were asked to look at 3 series of images on a screen. Images showed a virtual supermarket aisle. The aisle had two identical opposite gondolas, each containing the same number of items of same size and shape. In each image, apart from one single colored item, all other items were gray. In each series, the colored item was always of the same primary color (blue, red or green) and it was displayed an equal number of times on the right and on the left. For each image, subjects were asked to locate the colored item as quickly as possible. Accuracy and response time of answers were recorded. Colored items were perceived more accurately and rapidly when they were displayed on the left gondola to the observer. This phenomenon was specifically color-dependent, as red and blue items were better perceived than green ones. Our results support the hypothesis of an asymmetric perception of colored items in the aisle. Retailers should consider that the consumer's perception of colored package is more accurate and fast when the items are positioned on the left gondola. They should also consider that some colors may be more effective than others in catching customers' attention.
\end{abstract}

Keywords: Positioning of Packages, Color Perception, Virtual Aisle, Brain Laterality Theory

\section{INTRODUCTION}

The existence of an asymmetric perception of verbal and non-verbal package design components was described in the pioneering work by Rettie and Brewer (2000). By performing rapid visual perception experiments designed to simulate the real conditions of product purchase, these authors showed that verbal elements of packaging were distinguished more frequently and correctly when they were on the left side of the package (i.e., to the right of the viewer). Conversely, non-verbal elements were perceived significantly more accurately when they were on the right (i.e., to the left of the viewer). The two authors explained these results on the basis of the Theory of brain laterality. According to this theory the two human cerebral hemispheres, which are only approximately symmetrical in terms of anatomy, are functionally specialized and lateralized with regards to a number of different cognitive processes (Hugdahl, 2000). Furthermore, Rettie and Brewer (2000) proposed that the Theory of brain laterality might also be applied to other aspects of merchandising and particularly to the positioning of packs relative to Corresponding Author: Daniele Porcheddu, Department of Economics and Business, University of Sassari, Via Muroni 25, 07100 Sassari, Italy Tel: +39(0)79213001 Fax: +39(0)79213002 
traffic flow in stores and concluded that products on the right-hand side of the aisle would be expected to sell better. However, they did not provide any experimental evidence to substantiate this assumption.

To the best of our knowledge, we are unaware of any previously reported study exploring, in an experimental framework of rapid perception, the presence of a possible perceptual asymmetry of non-verbal stimuli displayed in a context simulating a modern shopping aisle.

The objective of this study is to confirm (or confute), in a lab setting, some of the implications of Rettie and Brewer's (2000) study. More specifically, our study aims to evaluate the existence of a possible perceptive asymmetry of individuals with respect to a specific family of non-verbal stimuli (these colors) placed in a context that simulates an aisle of a retail outlet.

The questions we want to answer are the following: is the color perception of packages in a supermarket affected by: (a) their in-aisle position? (b) their color type?; and (c) an interaction of the in-aisle position and the color type?

\section{MATERIALS AND METHODS}

The tests were carried out in November 2011 in a store of a leading European supermarket chain. Shoppers passing by a detection position we set up near the information booth, were contacted and notified about the actual experiment. One hundred and twenty healthy, right-handed subjects, recruited on a voluntary basis, took part in this survey. The sample size is sufficient to vouch for the main effect studied (i.e., the in-aisle position), with $\alpha=0.05$ : (a) an a priori power of $95 \%$; (b) an effect size of at least moderate intensity $\left(\eta^{2}=\right.$ 0.059) (Clark-Carter, 2004; Faul et al., 2007). Inclusion criteria were: (1) best corrected visual acuity $\geq 20 / 20$ in each eye; (2) normal color vision, determined by Ishihara color plates; (3) right-handedness and laterality quotient, assessed by an extended version of the Edinburgh Handedness Inventory (Cohen, 2008; Oldfield, 1971) no history of ophthalmic and/or neurological disorders.

Subjects were asked to sit in front of a 50-inch plasma screen with $1,920 \times 1,080$ pixel resolution and 100 $\mathrm{Hz}$ refresh rate, at a distance of $55 \mathrm{~cm}$, with their chins resting on a chin-rest (taken from a slit-lamp) and look at three series of 18 images each. Pictures were presented in a random sequence to prevent possible order effects on the results. The images showed a virtual modern store aisle, as it would be seen by a customer standing at one end in a central position and looking straight ahead (Fig. 1).

On both sides, the virtual aisle featured a gondola (this term refers to a system of shelving which offers stacked merchandise to the customer in a longitudinal presentation, see Varley, 2006) with five shelves, each containing 39 items of the same size and shape. In each image, apart from one single colored item, all other items were dark gray (HEX A9A9A9, RGB 169.169.169). In each series, the colored item was always of the same color, namely blue (HEX 0000FF, RGB 0.0.255), red (HEX FF0000, RGB 255.0.0), or green (HEX 00FF00; RGB 0.255.0) and it was displayed an equal number of times on the right and on the left. All colors were monochromatic and had the same level of saturation. The selection of these primary colors was made in accordance with the dichromatic theory of human color vision, also known as Young-Helmholtz Theory. According to this theory, the human eye has three types of cones (each one characterized by a particular pigment) having a different spectral sensitivity (with peaks in different spectral regions of light). In particular, we distinguish $\mathrm{S}$ cones (with maximum sensitivity to blueviolet), $\mathrm{M}$ cones (especially sensitive to green) and $\mathrm{L}$ cones (very sensitive to red). The colors used were placed near the peak sensitivity of cones of their respective family.

To avoid perceptual bias, all the images shown were perfectly symmetrical to the vertical axis. The average item dimensions were $0.76^{\circ}$ horizontally and $1.04^{\circ}$ vertically, when seen from a distance of $55 \mathrm{~cm}$. At the beginning of the experiment, a sample image was shown for $10 \mathrm{sec}$. Then, each subject was asked to stare at a central white cross on a black background (fixation cross). After $3 \mathrm{sec}$, the first image was shown for 150 msec. Then, the fixation cross re-appeared for $3 \mathrm{sec}$, followed by a new test image for $150 \mathrm{msec}$. The same procedure was repeated until completion of the experiment (Fig. 2).

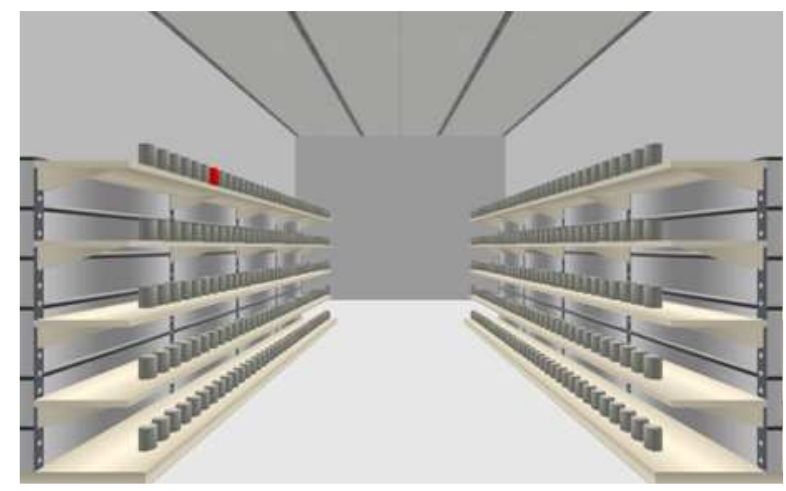

Fig. 1. Image showing a virtual modern store aisle with a colored item in the left gondola 


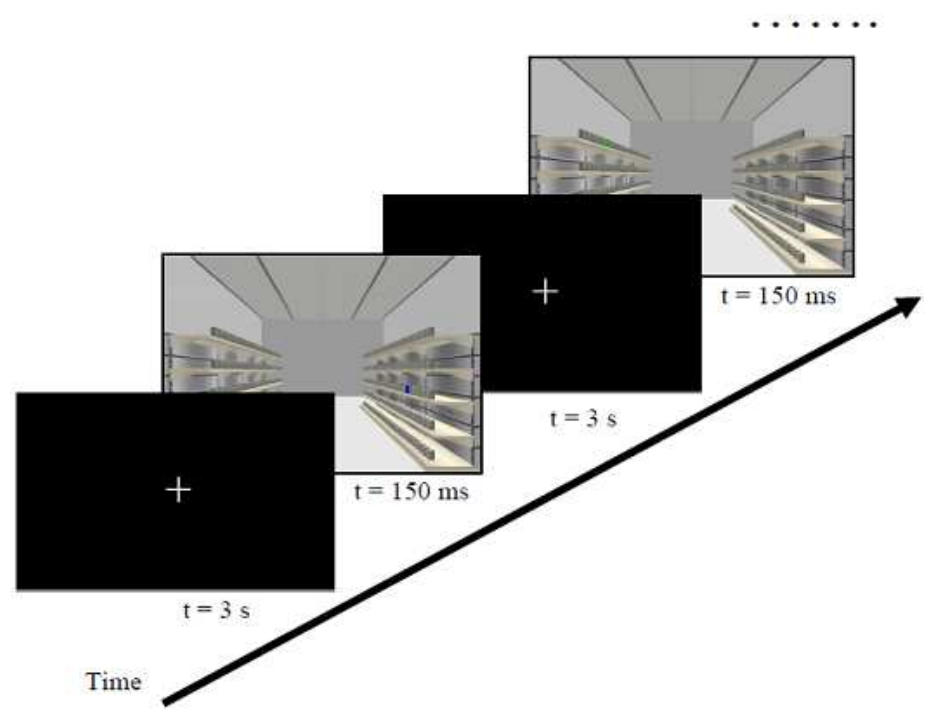

Fig. 2. Sequence of stimuli and experimental timeline

Table 1. Perception of colored items in the right and left visual hemifields: response accuracy $(\%)$ and the associated average response times (ms) Colored item position

Left visual field

Right visual field Response accuracy $(\%) \pm$ S.D. ${ }^{\text {a }}$

Blue Green Red

$\begin{array}{lll}96 \pm 6 & 88 \pm 14 & 98 \pm 5\end{array}$
$92 \pm 12$ $86 \pm 15$ $93 \pm 9$

\section{Average response times $(\mathrm{ms}) \pm$ S.D. ${ }^{\mathrm{a}}$}

Blue

$\begin{array}{ll}294 \pm 134 & 376 \pm 176 \\ 358 \pm 147 & 402 \pm 147\end{array}$ $402 \pm 147$
For each image, subjects were asked to locate as quickly as possible the colored item and immediately click on the right or left key of a response pad (model RB-530, Cedrus Corporation San Pedro, CA) to indicate the location of the colored item (on the right or left, respectively). SuperLab software (version 4.0.7, Cedrus Corporation, San Pedro, CA) was used for visual stimuli presentation. Correctness and response time of answers were recorded.

Statistical analysis was performed by two-factor repeated measures ANOVA, with visual hemifield (right or left) and color (green, blue, or red) both as withinsubjects factors.

\section{RESULTS}

One hundred and twenty subjects (60 men, 60 women; mean age: 40.2 \pm 14.3 years; range $15-69$ years) participated in the survey. The average laterality quotient was 86.11 \pm 13.63 (range 53.3-100) (Cohen, 2008; Oldfield, 1971). A total of 12,960 observations were processed 6,480 answers and 6,480 response times).

The percentage of response correctness and the average response times are summarized in Table $\mathbf{1 .}$

On the whole, irrespective of colors, colored items were perceived significantly more accurately and rapidly when they were displayed on the left of the observer.

ANOVA for response correctness highlighted the significance of both the main effect relative to the visual hemifield factor $\left[\mathrm{F}(1,119)=27.831, \mathrm{p}<0.001\right.$ (partial $\eta^{2}$ $=0.190)]$ and the effect relative to the color factor $[\mathrm{F}(2$, $238)=38.727, \mathrm{p}<0.001\left(\right.$ partial $\left.\left.\eta^{2}=0.246\right)\right]$. However, the effect of visual hemifield $\times$ color interaction $[\mathrm{F}(2$, $238)=1.709, p=0.183$ ] did not appear to be relevant. Post hoc tests with Bonferroni corrections showed that red and blue stimuli, when compared with green ones, were perceived significantly more accurately $(\mathrm{p}<0.001)$; on the other hand, there was no statistically significant difference in response correctness when comparing blue and red stimuli.

ANOVA for response times showed the significance of both the main effect relative to the visual hemifield factor $\left[F(1,119)=29.445, p<0.001\left(\right.\right.$ partial $\left.\left.\eta^{2}=0.198\right)\right]$ and the effect relative to the color factor $[(2,238)=$ $29.278, \mathrm{p}<0.001$ (partial $\eta^{2}=0.197$ )]. The effect of visual hemifield $\times$ color interaction $[\mathrm{F}(2,238)=2.239, \mathrm{p}=$ 0.109 ] was not found to be significant. Post hoc tests with Bonferroni corrections disclosed that red and blue stimuli, when compared with green ones, were perceived 
rapidly $(\mathrm{p}<0.001)$. Conversely, no statistically significant difference in response times was found, when blue and red stimuli were compared.

\section{DISCUSSION}

In this study on the perception of colored items in the aisle of a virtual store, we found that items are perceived more accurately and rapidly when they are displayed on the left-hand side of the aisle.

Former research has demonstrated that verbal and non-verbal components of package design are perceived differently (Rettie and Brewer, 2000): Verbal elements of packaging were distinguished significantly more frequently and correctly when they were displayed on the right of the viewer; on the other hand, non-verbal elements were perceived significantly more accurately when they were displayed on the left of the observer. These results were explained on the basis of the functional features of the two human cerebral hemispheres, which, despite being anatomically approximately symmetrical, are functionally specialized and lateralized with regards to a number of different cognitive processes. According to the most common interpretation of this phenomenon, hemispheric specialization can be reduced to two patterns-one linguistic, the other visual-spatial (Hugdahl, 2000; 2005; Jancke and Steinmetz, 2004). In particular, in righthanded individuals, the left hemisphere is thought to be specialized in processing verbal stimuli, whereas the right hemisphere essentially deals with non-verbal stimuli. However, some authors have criticized the validity of this generally accepted hemispheric specialization pattern (Bertelson, 1981; Hellige, 1996).

In their study on verbal and visual components of package design, Rettie and Brewer (2000) suggested that the Theory of brain laterality can be applied to other aspects of merchandising. Even though their study was focused on the perception of verbal and non-verbal components of product package design, they inexplicably extended their conclusions to the positioning of products in supermarket aisles and stated that products on the righthand side of the aisle would be expected to sell better. As a matter of fact, the authors failed to show any experimental evidence to justify their conclusion.

Despite the total lack of experimental evidence, the implications suggested by Rettie and Brewer (2000) were taken for granted by two later studies (Larson, 2006; Grimes, 2006).

Indeed, in a recent article on supermarket aisle management, Larson (2006) stated that if most shoppers follow the same traffic pattern, stores may better communicate messages by having pictorial or visual signs on the left-hand side of the aisle and text signs on the right. Furthermore, according to Grimes (2006), the positioning of packs in stores may also be subject to hemispheric processing effects, with products on the right-hand side of the aisle selling better. Surprisingly enough, neither study contains any specifically designed research, or even quotations from former reports, to justify applying Rettie and Brewer's conclusions to the problem of item positioning in relation to traffic flow in stores.

There are some recent reports which have tested the applicability of brain laterality Theory to marketing and merchandising. For example, Gontijo et al. (2002) investigated the category of brand names and suggested that they have a special neuropsychological status. These authors found that "common nouns, brand names, weird non-words and normal non-words" were recognized in this decreasing order of speed and accuracy. A right visual field advantage was disclosed for all four lexical types; however, brand names, like non-words, were found to be less lateralized than common nouns, consistent with theories of categoryspecific lexical processing. In another survey, Coulter (2002) showed that higher than expected demand associated with 9-ending prices is more likely when prices are placed to the left of attended verbal information contained in an advertisement.

We are unaware of any previously reported study addressing the problem of color perception of packs relatively to their in-aisle position and adopting an experimental framework simulating a context of rapid perception. The recent study by Porcheddu et al. (2011) effectively tackled the phenomenon of asymmetric perception of some non-verbal stimuli located in an aisle of a virtual store but using different experimental conditions.

In our virtual model, based on the presentation of rapid visual stimuli, we found that colored items displayed on the shelves of a virtual store were distinguished significantly more correctly and quickly when they were presented on the left-hand side of the aisle. This phenomenon was specifically colordependent, as red and blue items were perceived more accurately and rapidly than green ones. Overall, our results confirm that non-verbal elements, like the colored stimuli, are seen more accurately and rapidly when they are displayed on the left of the observer, as originally postulated by Rettie and Brewer (2000).

Generally speaking, positioning a product on the left-hand rather than on the right-hand shelves may 
induce differences in the accuracy and speed of visual perception by customers standing at one end of an aisle. It also seems reasonable to expect that this perceptual asymmetry still persists even when the subject is halfway along the aisle.

\section{MANAGERIAL IMPLICATIONS AND MAIN LIMITATIONS OF THE EXPERIMENT}

Dreze et al. (1994) noted that: "The retailing environment is very noisy, with hundreds of competing stimuli vying for attention". Our study showed that some item characteristics may have an important role in the process of visual perception that can lead the item to be noted.

\subsection{Perceptual Asymmetries Between Different in-Aisle Positions}

The literature has highlighted how some shelf positions are more attractive than others. For example, as far as the vertical dimension is concerned, there is general agreement about the existence of some more desirable locations, which are generally found at eyelevel and/or mid-shelf (Valenzuela and Raghubir, 2009; Chandon et al., 2009; Porcheddu and Venturi, 2011). Shelf locations and associated shelf position effects (Dreze et al., 1994) can be defined as being of an intragondola type. Our study leads us to believe in the existence of another family of in-aisle positions and associated effects of an inter-gondola type, relating to the importance of placing the reference on a given gondola rather than on that which is opposite. The results of our study suggest that retailers should take into account the fact that, at least with respect to colored stimuli and a hypothetical traffic pattern, positioning on the left rather than on the right of the aisle, is not indifferent in terms of accuracy and speed of perception by consumers.

\subsection{Perceptual Asymmetries Between Colors}

Furthermore, the statistical significance of differences between the percentage of correct responses and RTs relatively to the different colors is suggestive of a sort of intra-family dependence of the results obtained during the experiment: with all other conditions identical, the perception of a blue or red package seems to be more accurate and faster than the perception of a green one. These results are important in terms of package design, because they demonstrate how some colors may be more effective than others in bringing customers' attention.
In general, as stated by Masson and Wellhoff (2005), the best known rule of merchandising is "not seen not taken, not taken not sold". Actually, item perception is the first essential step in a neuropsychological process that can lead (obviously in a non-deterministic way) the item to be chosen.

\subsection{Limitations and Future Research}

Our study has several limitations. First of all, we used a virtual model, which does not reflect exactly the real situation in a supermarket aisle. Furthermore, our results are clearly restricted to the family of colored stimuli. Last but not least, our study was limited to righthanded subjects.

Further experimental studies are necessary for a better understanding of the mechanism by which products are perceived in a supermarket aisle. Future research may use more realistic laboratory situations (e.g., 2-D pictures associated with 3-D eyewear/visors/helmets or purpose-built experimental stations physically simulating a supermarket aisle) and assess other categories of non-verbal stimuli (singularly or simultaneously considered).

\section{ETHICAL ASPECTS}

The authors have no financial interest in any material used in this research.

The subjects received detailed information and signed an informed consent form for participation in the experiment, which was therefore voluntary. No compensation whatsoever was offered to the participants.

\section{ACKNOWLEDGMENT}

The authors thank Myriam D'Assunta, Luca Ferrucci and Gianpiero Lugli for their valuable suggestions. The usual disclaimer applies.

\section{REFERENCES}

Bertelson, P., 1981. The nature of hemispheric specialization: Why should there be a single principle? Behav. Brain Sci., 4: 63-64. DOI: 10.1017/S0140525X00007561

Chandon, P., J.W. Hutchinson, E.T. Bradlow and S.H. Young, 2009. Does in-store marketing work? Effects of the number and position of shelf facings on brand attention and evaluation at the point of purchase. J. Market., 73: 1-17. DOI: 10.1509/jmkg.73.6.1 
Clark-Carter, D., 2004. Quantitative psychological research: A Student's Handbook. 2nd Edn., Psychological Press, New York, ISBN-10: 1841692255, pp: 649.

Cohen, M.S., 2008. Handedness questionnaire.

Coulter, K.S., 2002. The influence of print advertisement organization on odd-ending price image effects. J. Prod. Brand Manage., 11: 319-334. DOI: 10.1108/10610420210442193

Dreze, X., S.J. Hoch and M.E. Purk, 1994. Shelf management and space elasticity. J. Retailing, 70: 301-326. DOI: 10.1016/0022-4359(94)90002-7

Faul, F., E. Erdfelder, A.G. Lang and A. Buchner, 2007. G*Power 3: A flexible statistical power analysis program for the social, behavioral and biomedical sciences. Behav. Res. Methods, 39: 175-191. PMID: 17695343

Gontijo, P.F.D., J. Rayman, S. Zhang and E. Zaidel, 2002. How brand names are special: Brands, words and hemispheres. Brain Language, 82: 327-343. DOI: $10.1016 / \mathrm{S} 0093-934 \mathrm{X}(02) 00036-6$

Grimes, A., 2006. Are we listening and learning? Understanding the nature of hemispherical lateralisation and its application to marketing. Int. J. Market Res., 48: 439-458.

Hellige, J.B., 1996. Hemispheric Asymmetry for Components of Visual Information Processing. In: Brain Asymmetry. Davidson, R.J. and K. Hugdahl, (Eds.). MIT Press, Cambridge, ISBN-10; 0262540797, pp: 99-122.

Hugdahl, K., 2000. Lateralization of cognitive processes in the brain. Acta Psychol., 105: 211-235. DOI: 10.1016/S0001-6918(00)00062-7

Hugdahl, K., 2005. Symmetry and asymmetry in the human brain. Eur. Rev., 13: 119-133. DOI: $10.1017 / \mathrm{S} 1062798705000700$

Jancke, L. and H. Steinmetz, 2004. Anatomical Brain Asymmetries and Their Relevance for Functional Asymmetries. In: The Asymmetrical Brain, Hugdahl, K. and R.J. Davidson (Eds.). MIT Press, Cambridge, MA., ISBN-10: 0262582546, pp: 187-229.
Larson, R., 2006. Core principles for supermarket aisle management. J. Food Distribution Res., 37: 107-111.

Masson, J.E. and A. Wellhoff, 2005. Le Merchandising. 6th Edn., Dunod, Paris, ISBN: 9782100489022, pp: 360.

Oldfield, R.C., 1971. The assessment and analysis of handedness: The edinburgh inventory. Neuropsychologia, 9: 97-113. DOI: 10.1016/00283932(71)90067-4

Porcheddu, D. and A. Venturi, 2011. Choices from identical options in a virtual shopping aisle. Open Bus. J., 4: 36-45. DOI: 10.2174/1874915101104010036

Porcheddu, D., A. Usai and A. Venturi, 2011. Nonverbal stimuli in a virtual aisle: Does the shelf you choose matter? Int. Rev. Retail, Distribution Consumer Res., 21: 215-231. DOI: 10.1080/09593969.2011.578797

Rettie, R. and C. Brewer, 2000. The verbal and visual components of package design. J. Prod. Brand Manage., $\quad 9$ : $56-70 . \quad$ DOI: 10.1108/10610420010316339

Valenzuela, A. and P. Raghubir, 2009. Position-based beliefs: The center-stage effect. J. Consumer Psychol., 19: 185-196. DOI: 10.1016/j.jcps.2009.02.011

Varley, R., 2006. Retail Product Management: Buying and Merchandising. 2nd Edn., Routledge, London, ISBN-10: 0415327156, pp: 263. 